\title{
The association of endothelial injury and systemic inflammation with perioperative myocardial infarction
}

\section{Ollila, Aino}

$2019-11$

Ollila, A, Vikatmaa , L, Virolainen, J , Nisula , S , Lakkisto , P, Vikatmaa, P , Tikkanen , I, Venermo , M \& Pettila , V 2019 , ' The association of endothelial injury and systemic inflammation with perioperative myocardial infarction ' , Annals of Clinical Biochemistry , vol. 56 , no. 6 , 0004563219873357 , pp. 674-683 . https://doi.org/10.1177/0004563219873357

http://hdl.handle.net/10138/319730

https://doi.org/10.1177/0004563219873357

unspecified

publishedVersion

Downloaded from Helda, University of Helsinki institutional repository.

This is an electronic reprint of the original article.

This reprint may differ from the original in pagination and typographic detail.

Please cite the original version. 


\title{
The association of endothelial injury and systemic inflammation with perioperative myocardial infarction
}

\author{
Aino Ollila' (D, Leena Vikatmaa', Juha Virolainen², Sara Nisula', Päivi Lakkisto ${ }^{3,4}$, \\ Pirkka Vikatmaa $^{5}$, Ilkka Tikkanen ${ }^{4,6,7}$, Maarit Venermo ${ }^{5}$ and Ville Pettilä'
}

\begin{abstract}
Background: Major surgery predisposes to endothelial glycocalyx injury. Endothelial glycocalyx injury associates with cardiac morbidity, including spontaneous myocardial infarction. However, the relation between endothelial glycocalyx injury and the development of perioperative myocardial infarction remains unknown.

Methods: Fifteen perioperative myocardial infarction patients and 60 propensity-matched controls were investigated in this prospective study. The diagnosis of perioperative myocardial infarction was based on repeated cardiac troponin $\mathrm{T}$ measurements, electrocardiographs and recordings of ischaemic signs and symptoms. We measured endothelial glycocalyx markers - soluble thrombomodulin, syndecan-I and vascular adhesion protein I - and an inflammatory marker, namely interleukin-6, preoperatively and $6 \mathrm{~h}$ and $24 \mathrm{~h}$ postoperatively. We calculated the areas under the receiver operating characteristics curves (AUCs) to compare the performances of the different markers in predicting perioperative myocardial infarction. The highest value of each marker was used in the analysis.

Results: The interleukin- 6 concentrations of perioperative myocardial infarction patients were significantly higher preoperatively and 6 and $24 \mathrm{~h}$ postoperatively $(P=0.002, P=0.002$ and $P=0.001$, respectively). The AUCs $(95 \%$ confidence intervals) for the detection of perioperative myocardial infarction were 0.51 (0.34-0.69) for soluble thrombomodulin, $0.63(0.47-0.79)$ for syndecan-I, $0.54(0.37-0.70)$ for vascular adhesion protein I and $0.69(0.54-0.85)$ for interleukin-6.

Conclusions: Systemic inflammation, reflected by interleukin-6, associates with cardiac troponin $T$ release and perioperative myocardial infarction. Circulating interleukin-6 demonstrated some potential to predict perioperative myocardial infarction, whereas endothelial glycocalyx markers did not.
\end{abstract}

\section{Keywords}

Cardiology, analytical systems, proteins

Accepted: 6th August 2019

\footnotetext{
'Department of Anesthesiology, Intensive Care and Pain Medicine, University of Helsinki and Helsinki University Hospital, Helsinki, Finland ${ }^{2}$ Heart and Lung Center, University of Helsinki and Helsinki University Hospital, Helsinki, Finland

${ }^{3}$ Department of Clinical Chemistry and Hematology, University of Helsinki and Helsinki University Hospital, Helsinki, Finland

${ }^{4}$ Minerva Foundation Institute for Medical Research, Helsinki, Finland

${ }^{5}$ Department of Vascular Surgery, University of Helsinki and Helsinki University Hospital, Helsinki, Finland
}

${ }^{6} \mathrm{Helsinki}$ Hypertension Centre of Excellence, University of Helsinki and Helsinki University Hospital, Helsinki, Finland

${ }^{7}$ Abdominal Center Nephrology, University of Helsinki and Helsinki University Hospital, Helsinki, Finland

Corresponding author:

Aino Ollila, Biomedicum Helsinki 2C, Tukholmankatu 8 C, Fl-00290 Helsinki/P.O. Box 705, Helsinki 00029, Finland.

Email: aino.ollila@helsinki.fi 


\section{Introduction}

Major surgery causes sympathoadrenal activation and systemic inflammation, exposing the organ system to ischaemia-reperfusion ( $\mathrm{I} / \mathrm{R})$ injury. In addition, extensive perioperative fluid management may contribute to endothelial injury. The vascular endothelium represents the main organ affected by systemic inflammation, $\mathrm{I} / \mathrm{R}$ injury and hypervolemia, leading to endothelial activation and endothelial glycocalyx (EG) injury. ${ }^{1-5}$ After early endothelial distress, circulating EG components (syndecan-1) and endothelial cell integral membrane proteins (soluble thrombomodulin [sTM] and vascular adhesive protein [VAP-1]) are released into plasma and have been suggested to be sensitive markers of endothelial damage and potentially imminent organ injury. ${ }^{6}$ Pro-inflammatory cytokine IL-6 is central in acutephase reactions caused by infection or tissue trauma. ${ }^{7}$

The important role of EG injury has been established in numerous critical illnesses, including sepsis and acute kidney injury (AKI), and it is associated with increased mortality. ${ }^{8-10}$ Furthermore, EG injury associates with cardiovascular morbidity, including acute coronary syndrome (ACS). ${ }^{11-13}$ However, no data exist on the association of EG injury with perioperative myocardial infarction (PMI).

The objective of this study was to investigate the relation of EG injury and systemic inflammation with perioperative myocardial injury/infarction, evaluated by serial measurements of EG markers and IL- 6 . We hypothesized that acute inflammation and EG injury are associated with perioperative cardiac troponin $\mathrm{T}(\mathrm{cTnT})$ release and PMI.

\section{Methods}

\section{Study design}

This was a laboratory substudy of a prospective, observational single-centre study investigating the incidence of major perioperative cardiac events in patients undergoing systematic perioperative ischaemia screening, conducted at the Meilahti Hospital, Helsinki, Finland, in 2014. ${ }^{14}$ Ethical approval for the study (no. 11/13/03/ 02/2014) was provided by the Ethics Committee of the Department of Surgery at Helsinki University Hospital, Helsinki, Finland, on 30 January 2014. All patients or their next of kin signed a written informed consent before enrolment. In brief, we included 385 consecutive patients, aged 50 years or older, undergoing non-cardiac (vascular, thoracic or gastrointestinal) surgery between 3 April and 19 June 2014. Organ transplantations and hepatic surgery were excluded. In this substudy, we included all PMI patients with available baseline and follow-up blood samples $(n=15)$, as well as four propensity-matched controls for each PMI patient $(n=60)$. The variables used for matching were age, sex, main medical history, medications and intraoperative data.

\section{Patient management and data collection}

The procedure for obtaining the patients' medical history, demographics, medication, physiological and surgical data has been explained in detail previously. ${ }^{14}$ A blood sample for cTnT and an electrocardiograph (ECG) were obtained five times: preoperatively, $6 \mathrm{~h}$ from the conclusion of the operation, and on the first, second and third postoperative morning, or until discharge. Together with cTnT, we took $5 \mathrm{~mL}$ of EDTA plasma for a future analysis of endothelial EG markers and IL-6. Possible ischaemic signs and symptoms were obtained from the patients' charts and medical records filled in daily by the treating clinical staff. Based on repeated cTnT measurements, ECGs and clinical observations, a cardiologist (JV) blinded to the EG marker and IL-6 analyses confirmed all PMI diagnoses. The diagnosis of PMI was defined as a rise and/or a decline in cTnT, with at least one value above the upper reference limit (14 ng/L), and either ischaemic ECG changes or other ischaemic features present (symptoms of cardiac ischaemia, cardiac imaging evidence of myocardial infarction, autopsy findings of acute or healing myocardial infarction), as recommended. ${ }^{15}$

\section{Blood samples and enzyme-linked immunosorbent assay analyses}

Our focus was on perioperative EG injury, which is why only blood samples taken preoperatively and 6 and $24 \mathrm{~h}$ after surgery were analysed. cTnT concentrations were analysed immediately after sampling from a vein or an arterial catheter. We used a high-sensitivity fifthgeneration troponin $\mathrm{T}$ assay (electrochemiluminescense immunoassay [ECLIA]; Roche Diagnostics International Ltd, Rotkreuz, Switzerland) for the analyses. The limit of detection (LoD), analytical range, $10 \%$ coefficient of variation level and 99th percentile cut-off point were $5 \mathrm{ng} / \mathrm{L}, 5-10,000 \mathrm{ng} / \mathrm{L}, 13 \mathrm{ng} / \mathrm{L}$ and $14 \mathrm{ng} / \mathrm{L}$, respectively. Plasma samples were centrifuged at $22^{\circ} \mathrm{C}$ at $3170 \mathrm{r} / \mathrm{min}$ for $10 \mathrm{~min}$ within $3 \mathrm{~h}$ of sample collection. The centrifuged samples were stored in Mekamini $^{\mathrm{TM}}$ tubes (Mekalasi Oy, Nurmijäri, Finland) at $-80^{\circ} \mathrm{C}$ until all samples were collected for analysis. The biomarkers of EG injury (sTM, syndecan-1 and VAP-1) and the activation of the inflammatory system (IL-6) were analysed at the Minerva Foundation Institute for Medical Research, Helsinki, Finland, with commercially available immunoassays according to the manufacturers' recommendations: sTM (Quantikine ${ }^{\mathrm{TM}}$ 
ELISA, R\&D Systems Europe, Ltd, Abingdon, UK), $\mathrm{LoD} 0.0078 \mathrm{pg} / \mathrm{L}$, intra- and inter-assay coefficient of variation (CV) $2.3 \%-3.6 \%$ and $5.7-8.0 \%$ ); syndecan-1 (Human sCD138 [syndecan-1] ELISA Kit, Nordic BioSite AB. Täby, Sweden), LoD $0.0049 \mathrm{ng} / \mathrm{L}$, intraand inter-assay $\mathrm{CV} \quad 6.2 \%$ and $10.2 \%$, respectively; VAP-1 (Quantikine ${ }^{\mathrm{TM}}$ ELISA, R\&D Systems Europe, Ltd, Abingdon, UK), LoD $0.000024 \mathrm{ng} / \mathrm{L}$, intra- and inter-assay CV $1.5 \%-2.4 \%$ and $4.5 \%-4.8 \%$, respectively; IL-6 (Quantikine ${ }^{\mathrm{TM}}$ ELISA, R\&D Systems Europe, Ltd, Abingdon, UK), LoD $<0.0007 \mathrm{pg} / \mathrm{L}$, intra- and inter-assay CV $1.6 \%-4.2 \%$ and $3.3 \%-6.4 \%$, respectively. In one patient, the preoperative IL-6 value was under the LoD. All other endothelial and inflammatory markers were above the LoD.

\section{Outcomes}

To explore the association of EG injury and systemic inflammation, as reflected by IL-6, with cTnT release and PMI.

\section{Statistical analyses}

The assumption of a clinically significant two-fold increased level of laboratory parameters (sTM, syndecan-1, VAP-1, IL-6) in PMI patients compared with non-PMI patients and of a standard deviation equal to the difference would require a sample size of at least 10 patients and 40 controls (with a significance level of 0.05 and a power of $80 \%$ ). Based on these assumptions, we considered a total sample size of 75 patients (15 PMI patients and 60 propensity-matched non-PMI patients) to be adequate for comparisons between the groups. To match controls with PMI patients, we calculated a propensity score for each patient based on demographic and medical data. The variables included in the propensity matching are presented in supplemental digital content (S) Table 1. In brief, the variables included age, sex, main medical history, medications and intraoperative data. Four propensity-matched controls were selected for each PMI patient.

We present continuous variables as medians with interquartile ranges (IQRs) and dichotomous variables as absolute numbers with percentages. The comparisons of continuous variables and frequencies were made using the Mann-Whitney U-test and Fisher's exact test, respectively. We calculated the HodgesLehmann estimator with $95 \%$ CIs to measure the magnitude of differences between PMI patients' and non-PMI patients' preoperative, $6 \mathrm{~h}$ and $24 \mathrm{~h}$ postoperative values, as well as the highest values of EG markers and IL-6. We calculated the areas under the receiver operating characteristics curves (AUC) with
95\% CIs. The highest plasma concentrations of EG markers and IL-6 were used as test variables. We defined an AUC of $0.5-0.75$ as poor, $0.75-0.9$ as good and $>0.9$ as excellent, as suggested. ${ }^{16} \mathrm{~A}$ twosided $P$-value of $<0.05$ was considered significant. Statistical analyses were performed with SPSS version 23 (SPSS; Chicago, IL, USA).

\section{Results}

With regard to PMI, a total of 75 patients (15 with PMI and 60 matched controls) were included in the present study (Figure 1). In order to assess the generalizability of the current results, we compared the baseline characteristics of the primary study patients with all plasma samples taken to those with lacking plasma samples. The results are presented in Tables S1 and S2. With the exception of the differences in the numbers of urgent/emergency operations, the use of vasopressor support, the American Society of Anesthesiologists class and perioperative cardiac risk, ${ }^{17}$ the patients were comparable in terms of baseline characteristics.

\section{PMI}

The demographics, medical history and preoperative clinical findings of the PMI patients and their propensity-matched controls are summarized in Table 1. The 90-day mortality of PMI patients was $20 \%$, compared with the $8.3 \%$ among non-PMI patients.

Table 2 presents the median values of EG markers and IL-6 in patients with and without PMI. The PMI patients had higher IL-6 concentrations during the whole perioperative $24 \mathrm{~h}$ follow-up, but the sTM, syndecan-1 and VAP-1 concentrations were comparable in patients with and without PMI at all time points.

\section{The association of systemic inflammation and EG injury with perioperative $c T n T$ release}

Figure 2 illustrates the kinetics of the EG markers and IL-6 in patients with and without PMI. The IL-6 concentrations of PMI patients were significantly higher across all time points $-P=0.002, P=0.002$ and $P=0.001$, respectively. However, the sTM, syndecan1 and VAP-1 concentrations did not differ between PMI and non-PMI patients.

We tested the potential correlations between cTnT, IL-6 and EG markers in PMI and non-PMI patients with Spearman's rho. The correlations are presented in Tables S3A and S3B. The preoperative IL-6 concentration had a positive correlation with preoperative, $6 \mathrm{~h}$ postoperative and $24 \mathrm{~h}$ postoperative cTnT in PMI patients, $P=0.05$. Furthermore, in PMI patients, the $24 \mathrm{~h}$ postoperative sTM correlated positively with 
Table I. Characteristics of the PMI patients and propensity-matched controls.

\begin{tabular}{|c|c|c|c|}
\hline & $\begin{array}{l}\text { PMI } \\
N=15 \\
n(\%) \text { or } \\
\text { median [IQR] }\end{array}$ & $\begin{array}{l}\text { No PMI } \\
N=60 \\
n(\%) \text { or } \\
\text { median [IQR] }\end{array}$ & $\begin{array}{l}\text { All patients } \\
N=75 \\
n(\%) \text { or } \\
\text { median [IQR] }\end{array}$ \\
\hline Age & $75[65-86]$ & $70[62-76]$ & 71 [64-77] \\
\hline Gender (male) & $9(60)$ & $39(65)$ & $48(64)$ \\
\hline \multicolumn{4}{|l|}{ Comorbidity } \\
\hline CAD & $5(33.3)$ & $16(26.7)$ & $21(28)$ \\
\hline Heart failure & $4(26.7)$ & 7 (II.7) & II (I4.7) \\
\hline Baseline creatinine $>177 \mu \mathrm{mol} / \mathrm{L}$ & $2(13.3)$ & $2(3.3)$ & $4(5.3)$ \\
\hline ASO & $9(60)$ & $18(30)$ & $27(36)$ \\
\hline Hypertension & $8(53.3)$ & $39(65.0)$ & 47 (62.7) \\
\hline Diabetes & $2(13.3)$ & $19(31.7)$ & $21(28)$ \\
\hline COPD & $6(40)$ & $8(13.3)$ & 14 (I8.7) \\
\hline Current malignancy & 0 & $10(16.7)$ & $10(13.3)$ \\
\hline \multicolumn{4}{|l|}{ Prior } \\
\hline Acute MI & $4(26.7)$ & $9(15)$ & $13(17.3)$ \\
\hline $\mathrm{PCl} / \mathrm{CABG}$ & $3(20)$ & $10(16.7)$ & $13(17.3)$ \\
\hline Stroke/TIA & $4(26.7)$ & $16(26.7)$ & $20(26.7)$ \\
\hline \multicolumn{4}{|l|}{ ASA classification } \\
\hline II & 0 & $6(10)$ & $6(8)$ \\
\hline III & $9(60)$ & $34(56.7)$ & $43(57.3)$ \\
\hline $\mathrm{IV}-\mathrm{V}$ & $6(40)$ & $19(31.7)$ & $25(33.3)$ \\
\hline Perioperative cardiac risk score ${ }^{17}$ & $1.19[0.66-3.73]$ & $0.79[0.54-2.04]$ & $0.8 \mathrm{I}[0.6 \mathrm{I}-2.55]$ \\
\hline Urgent/emergency operation & II (73.3) & $37(61.7)$ & $48(64)$ \\
\hline Vasopressor load during the day of surgery $(\mathrm{mg})$ & $2.87[0.48-6.76]$ & $0.70[0.01-\mid .5 I]$ & $0.75[0.11-2.50]$ \\
\hline Nadir $\mathrm{Hb}$ during hospitalization (g/L) & $95[88-113]$ & $103[90-120]$ & $102[89-119]$ \\
\hline
\end{tabular}

PMI: perioperative myocardial infarction; IQR: interquartile range; CAD: coronary artery disease; ASO: atherosclerotic disease; COPD: chronic obstructive pulmonary disease; MI: myocardial infarction; PCI: percutaneous coronary intervention; CABG: coronary artery bypass grafting; TIA, transient ischemic attack; ASA: American Society of Anesthesiologists; Hb: haemoglobin concentration.

preoperative, $6 \mathrm{~h}$ postoperative and $24 \mathrm{~h}$ postoperative cTnT $(P=0.05)$. In non-PMI patients, the sTM and cTnT concentrations correlated positively preoperatively $(P<0.001), 6 \mathrm{~h}$ postoperatively $(P=0.001)$ and 24 h postoperatively $(P<0.001)$.

\section{The association of systemic inflammation and EG injury with PMI}

Figure 3 is a graphic representation of the performance of the EG markers and IL-6 in predicting PMI. The results of the analysis are summarized in Table 3 . None of the EG markers were predictive of PMI when the highest values were analysed. IL-6 predicted PMI with an AUC of 0.69 (95\% CI 0.54-0.85), $P=0.02$. The best cut-off value for IL-6 was $0.238 \mathrm{pg} / \mathrm{L}$, with a sensitivity of 0.53 , specificity of 0.85 and positive and negative likelihood ratios of 3.56 (95\% CI 1.65-7.65) and 0.55 (95\% CI 0.32-0.95), respectively.

\section{Discussion}

In this prospective laboratory study, we demonstrated that high IL-6 concentrations during the perioperative period were associated with cTnT release and PMI. Furthermore, a high perioperative IL-6 concentration may be predictive of PMI, whereas EG markers (sTM, syndecan-1 and VAP-1) were not.

The vascular endothelium is an active paracrine, endocrine and autocrine organ that is crucial in regulating vascular tone and maintaining vascular homeostasis. Moreover, previous observations indicate that endothelial dysfunction may represent a key early step in the development of atherosclerosis and that it is also involved in plaque progression and the occurrence of atherosclerotic complications. ${ }^{18,19}$ According to current knowledge, endothelial dysfunction may play a critical role in the development of ACS, as a dysfunctional endothelium causes a pro-inflammatory state that contributes to enhanced coronary plaque 


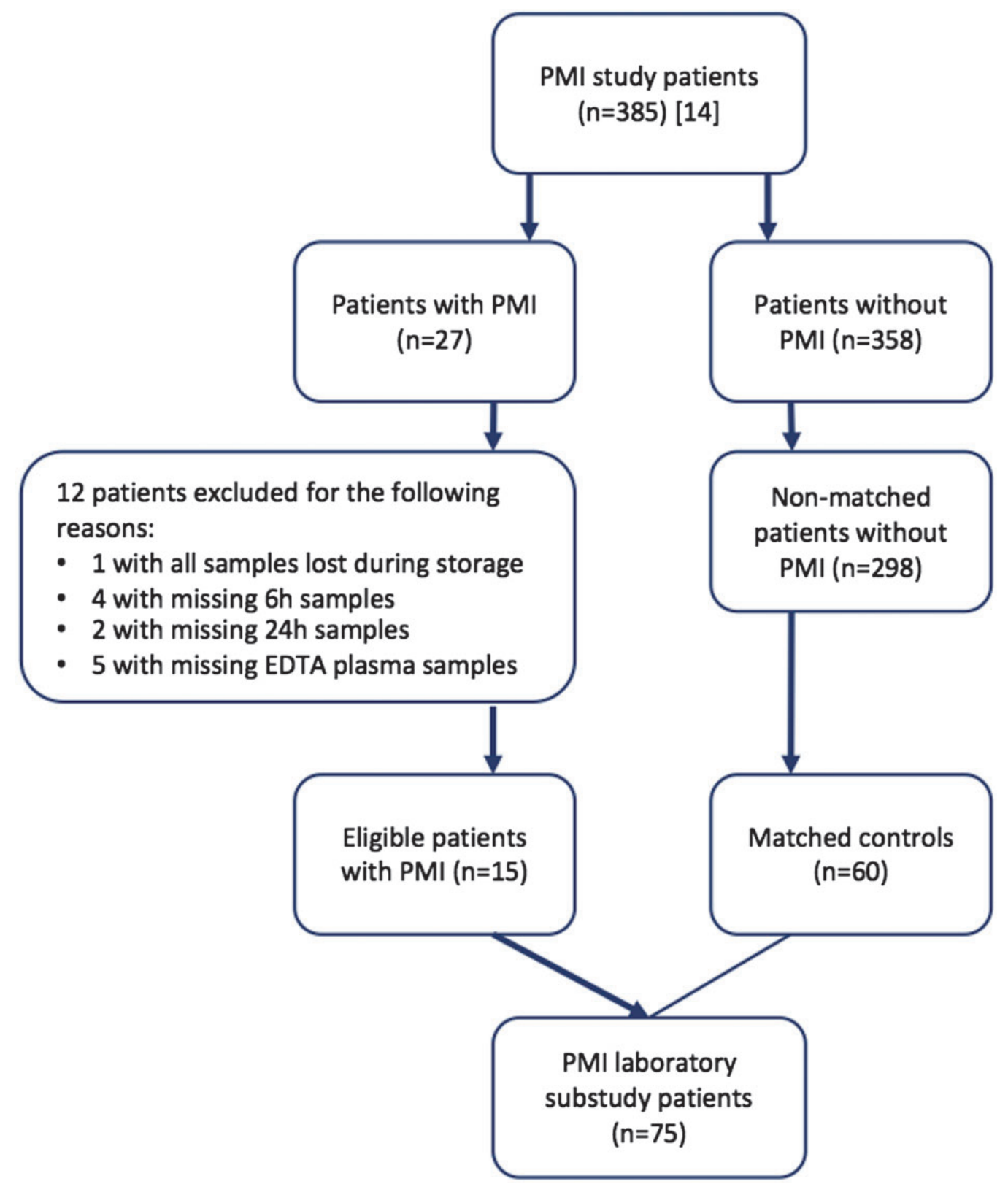

Figure I. Study flowchart.

PMI: perioperative myocardial infarction.

vulnerability. ${ }^{20}$ It also has a decreased ability to produce nitric oxide, resulting in vasospasm and impaired coronary arterial flow. ${ }^{20}$ Furthermore, endothelial dysfunction is characterized by a reduced anticoagulatory potential of the endothelium, an increased endothelial production of pro-coagulation mediators and an increased vasoconstriction due to platelet-derived mediators, ${ }^{11}$ resulting in a thrombogenic vascular environment.

Several studies have demonstrated the occurrence of EG injury in medical conditions characterized by sympathoadrenal activation, systemic inflammation and $\mathrm{I} / \mathrm{R}$ injury. ${ }^{8,10,21,22}$ Ostrowski et al. investigated patients with an acute ST segment elevation myocardial infarction (STEMI), admitted for primary percutaneous coronary intervention (pPCI) and demonstrated that these patients presented with high plasma concentrations of circulating STM, syndecan-1, adrenalin and noradrenalin. The authors showed that high circulating sTM was associated with myocardial injury as defined by high troponin I ( $\mathrm{TnI}$ ) release, and a similar tendency was seen in the current study (Tables S3A and S3B). 
Table 2. The median values of EG markers and IL-6 measured in PMI patients and propensity-matched controls.

\begin{tabular}{|c|c|c|c|c|}
\hline & $\begin{array}{l}\text { PMI } \\
n=I 5 \\
\text { median [IQR] }\end{array}$ & $\begin{array}{l}\text { No PMI } \\
n=60 \\
\text { median [IQR] }\end{array}$ & $P$ & $\begin{array}{l}\mathrm{HL} \\
(95 \% \mathrm{Cl})\end{array}$ \\
\hline \multicolumn{5}{|l|}{ Preoperative } \\
\hline sTM & $3.95[3.13-7.02]$ & 4.17 [3.47-4.74] & 0.87 & $-60.0(-10 \mid 4.0,751.0)$ \\
\hline Syndecan-I & $0.06[0.05-0.09]$ & $0.06[0.04-0.08]$ & 0.17 & $-9.0(-23.0,5.5)$ \\
\hline VAP-I & $0.36[0.30-0.5 \mathrm{I}]$ & $0.36[0.30-0.47]$ & 0.66 & $-13.5(-86.0,56.0)$ \\
\hline IL-6 & $0.03[0.01-0.09]$ & $0.007[0.003-0.03]$ & 0.008 & $-13.3(-27.3,-3.6)$ \\
\hline \multicolumn{5}{|c|}{$6 \mathrm{~h}$ postoperative } \\
\hline sTM & $3.50[2.94-5.25]$ & 4.00 [3.39-4.69] & 0.47 & $200.0(-501.0,846.0)$ \\
\hline Syndecan-I & $0.10[0.06-0.12]$ & $0.07[0.04-0.12]$ & 0.15 & $-13.4(-38.3,7.1)$ \\
\hline VAP-I & $0.32[0.25-0.38]$ & $0.33[0.26-0.42]$ & 0.57 & $18.0(-46.0,78.0)$ \\
\hline IL-6 & 0.21 [0.05-0.29] & $0.07[0.03-0.12]$ & 0.013 & $-72.1(-182.3,-12.2)$ \\
\hline \multicolumn{5}{|c|}{$24 \mathrm{~h}$ postoperative } \\
\hline sTM & $4.05[3.7 I-5.32]$ & 4.30 [3.65-5.07] & 0.94 & $44.5(-661.0,645.0)$ \\
\hline Syndecan-I & $0.07[0.05-0.1]$ & $0.07[0.05-0.09]$ & 0.44 & $-7.0(-24.4,11.2)$ \\
\hline VAP-I & $0.30[0.26-0.40]$ & $0.33[0.26-0.42]$ & 0.53 & $16.5(-35.0,74.0)$ \\
\hline IL-6 & $0.21[0.10-0.32]$ & $0.07[0.02-0.15]$ & 0.006 & $-93.2(-199.2,-23.1)$ \\
\hline \multicolumn{5}{|l|}{ Highest } \\
\hline sTM & $4.19[3.72-7.02]$ & 4.37 [3.65-5.34] & 0.87 & $-76.5(-920.0,646.0)$ \\
\hline Syndecan-I & $0.11[0.06-0.14]$ & $0.07[0.06-0.11]$ & 0.12 & $-19.1(-48.9,4.8)$ \\
\hline VAP-I & $0.38[0.30-0.5 \mathrm{I}]$ & $0.37[0.30-0.47]$ & 0.67 & $-15.5(-90.0,57)$ \\
\hline IL-6 & $0.24[0.10-0.35]$ & $0.10[0.04-0.19]$ & 0.022 & $-93.1(-213.0,-12.0)$ \\
\hline
\end{tabular}

IL-6: interleukin-6 (pg/L); EG: endothelial glycocalyx; PMI: perioperative myocardial infarction; HL: Hodges-Lehman estimator; IQR: interquartile range; Cl: confidence interval; sTM: soluble thrombomodulin (pg/L); syndecan-I (ng/L); VAP-I: vascular adhesion protein I (ng/L).

Moreover, high syndecan-1 concentrations were associated with short- and long-term mortality and heart failure. ${ }^{10}$ Sustained EG injury associates with the severity of post-cardiac arrest syndrome (PCAS), as was recently demonstrated by Bro-Jeppesen et al. ${ }^{22}$ The authors showed that baseline IL-6 concentrations were associated with EG activation and endothelial cell injury in patients with severe myocardial damage. ${ }^{22}$ However, to the best of our knowledge, no previous study exists concerning the association of endothelial injury with PMI.

The markers used to demonstrate EG activation and injury vary extensively between the studies. The different markers detected in circulating plasma represent the different phases of the endothelial activation and injury.

Firstly, we investigated thrombomodulin (TM), an integral membrane protein expressed on the surface of endothelial cells. It has a critical role as a cofactor in the protein $\mathrm{C}$ pathway that is important in regulating coagulation and inflammation. STM circulates in plasma and is found in increased concentrations in connection with various diseases, such as cardiovascular disease and diabetes, and in ischaemic or inflammatory endothelial damage. ${ }^{23}$ We found that the sTM concentrations correlated with cTnT release, especially in patients with no PMI. However, circulating STM was not predictive of PMI.

Secondly, we measured syndecan-1, an endothelial cell transmembrane protein, which exists in the form of proteoglycans. Previous animal studies suggest that the shedding of EG is associated with a significant increase in circulating syndecan-1, ${ }^{24,25}$ and this also appears to occur in humans during $\mathrm{I} / \mathrm{R}$ injury. ${ }^{9}$ In accordance with these findings, we observed a rise in syndecan- 1 concentrations when preoperative and $6 \mathrm{~h}$ postoperative values were compared. Like sTM, syndecan-1 was not predictive of PMI.

Thirdly, we studied VAP-1, which belongs to a family of semicarbamizide-sensitive amine oxidases (SSAOs) and is expressed in endothelial cells. VAP-1 plays an important role in controlling neutrophil adhesion to the endothelium and neutrophil extravasation from blood to tissues. Increased VAP-1 activity has been observed in critically ill patients with septic shock, ${ }^{26}$ and elevated concentrations have also been described in obese and diabetic persons. ${ }^{27}$ According to our findings, VAP-1 did not associate with 
(a)

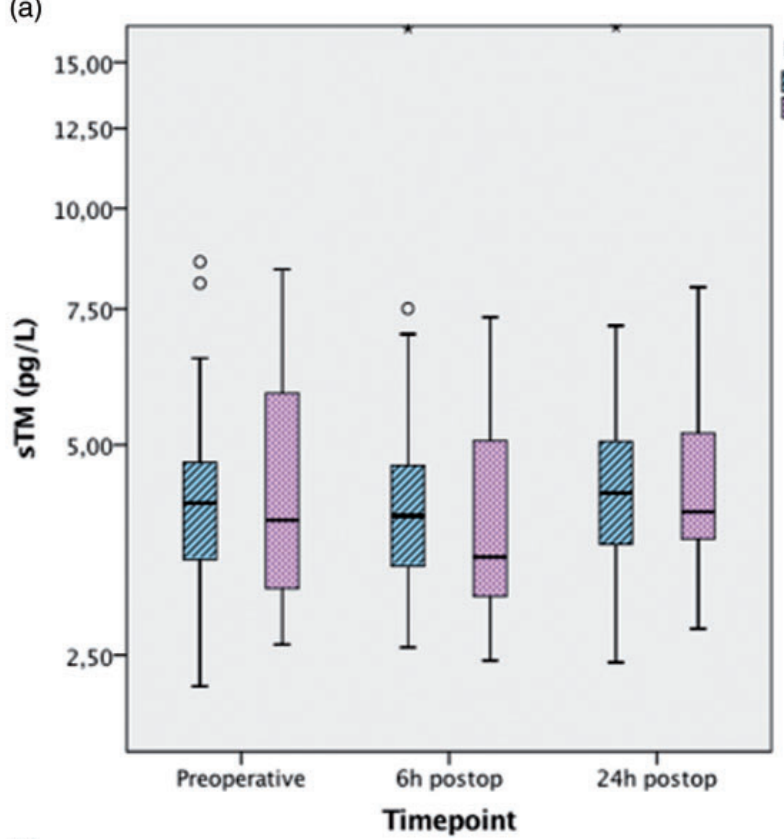

(c)

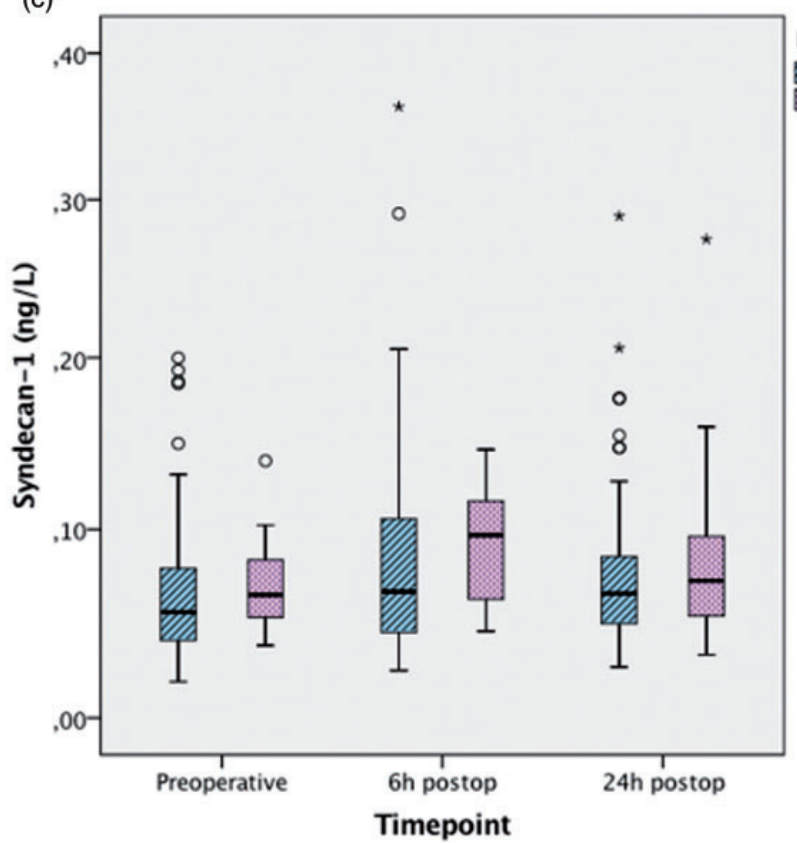

(b)

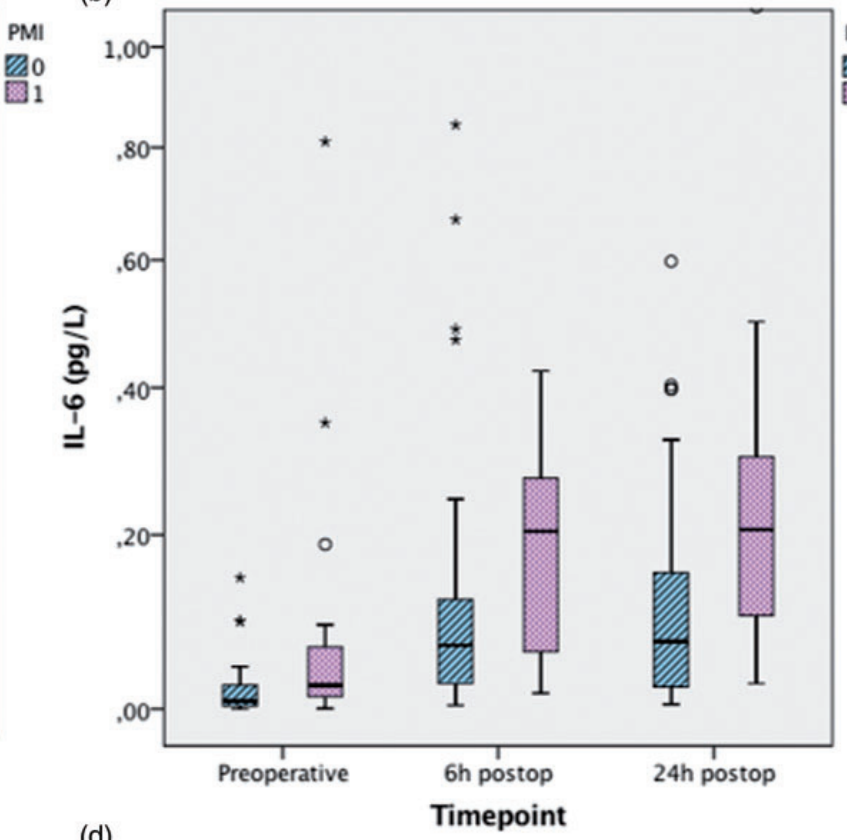

(d)

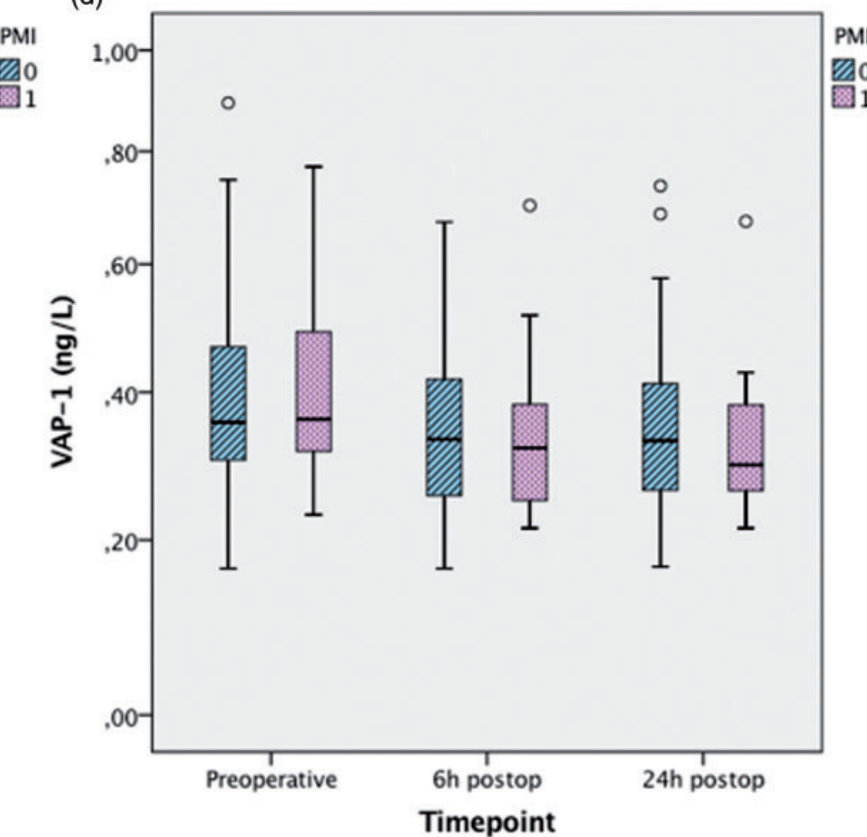

Figure 2. The kinetics of endothelial glycocalyx markers and interleukin-6 in PMI and non-PMI patients. Non-transformed values are presented on a logarithmic scale. Bars represent medians with interquartile ranges (IQRs). T-bars represent values I.5 times greater than the IQR.

PMI: perioperative myocardial infarction; sTM: soluble thrombomodulin; VAP-I: vascular adhesion protein I; IL-6: interleukin-6.

perioperative cTnT release or myocardial infarction. In addition to EG markers, IL-6 was measured as a proinflammatory cytokine produced mainly by macrophages and monocytes at an inflammatory site. IL-6 is a main stimulator in acute-phase reactions following an infection or tissue trauma but also plays an important role in the development of chronic inflammation. ${ }^{7}$
According to the current findings, a perioperative rise in IL-6 is associated with PMI and might be predictive of the complication.

The superiority of any endothelial marker in demonstrating endothelial injury in the clinical setting and in predicting the patients' outcome has not been demonstrated. In a perioperative setting, Rehm et al. 
(a)

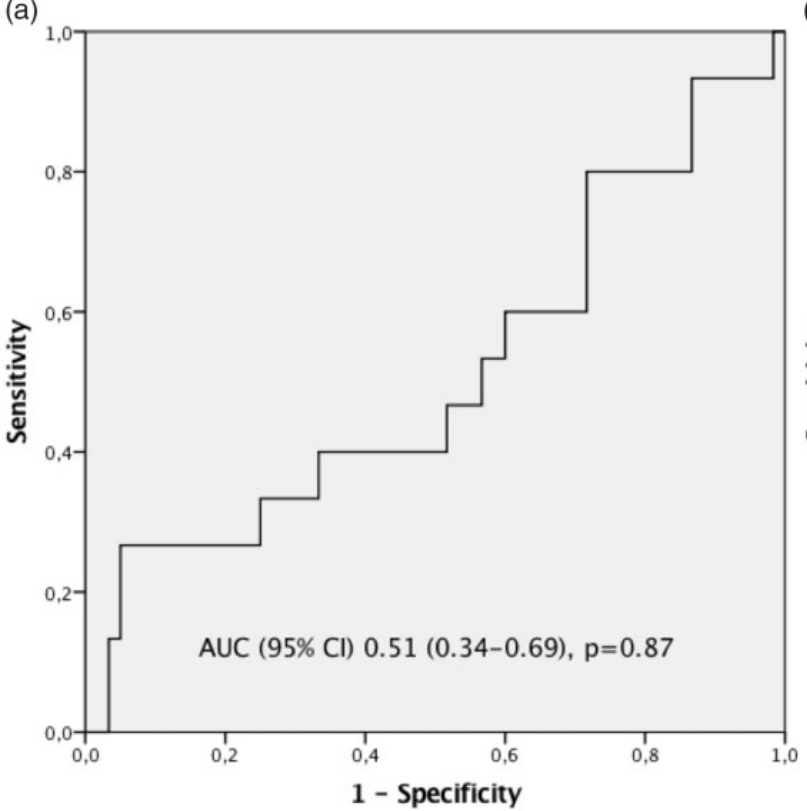

(c)

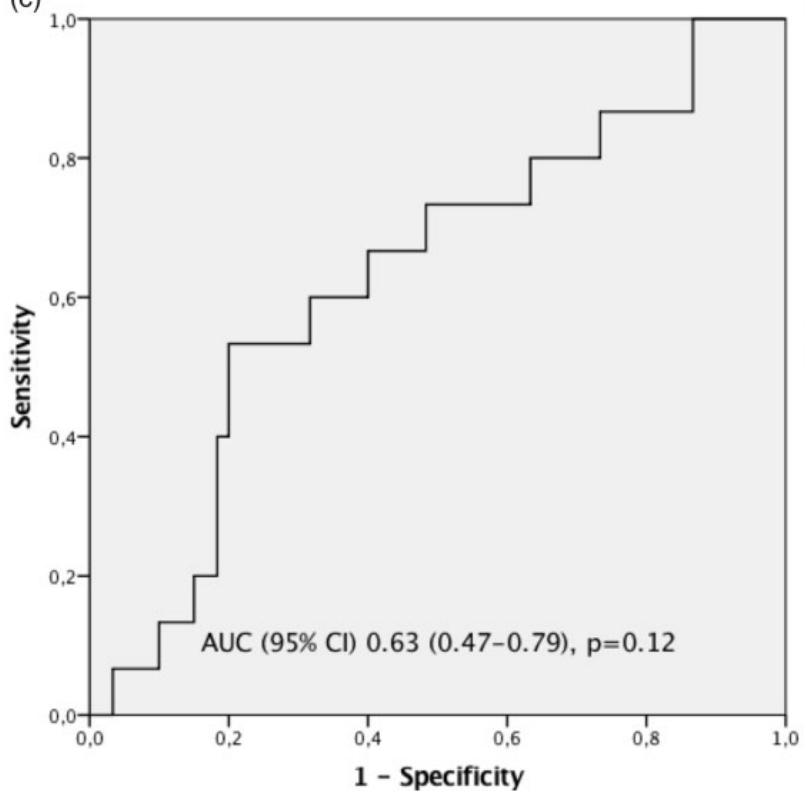

(b)

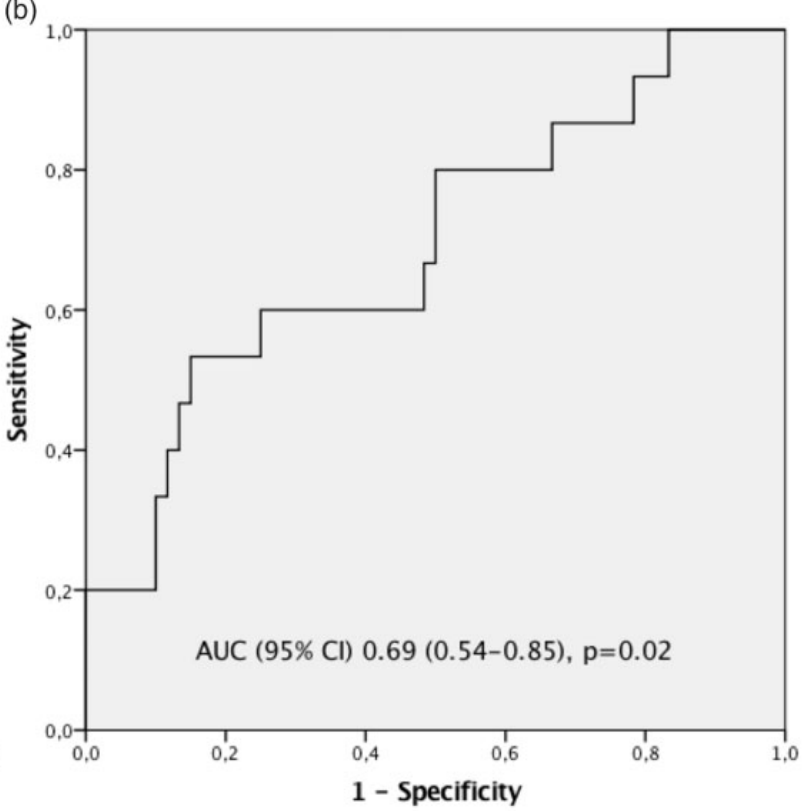

(d)

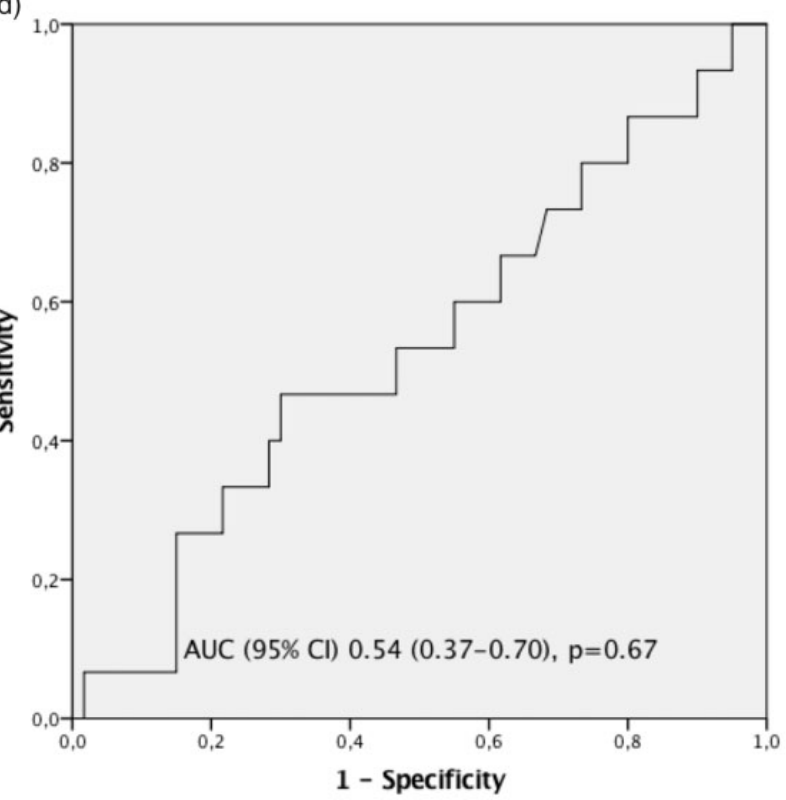

Figure 3. The highest values of the EG markers and IL-6 in predicting PMI. A: sTM, B: IL-6, C: syndecan-I, D: VAP-I. AUC: area under the receiver operating characteristics curve; $\mathrm{Cl}$ : confidence interval.

Table 3. The highest values of EG markers (sTM, syndecan-I and VAP-I) and IL-6 in predicting PMI.

\begin{tabular}{llllllllll}
\hline & & \multicolumn{3}{c}{ Cut-off } & & & & Youden \\
& AUC $(95 \% \mathrm{Cl})$ & value & Sensitivity & Specificity & LR+ (95\%Cl) & LR- (95\% CI) & index & PPV\% & NPV\% \\
\hline sTM & $0.51(0.34-0.69)$ & 6.83 & 0.27 & 0.95 & $5.33(1.33-21.33)$ & $0.77(0.57-1.05)$ & 0.22 & 57.1 & 83.8 \\
Syndecan-I & $0.63(0.47-0.79)$ & 0.11 & 0.53 & 0.80 & $2.67(1.33-5.33)$ & $0.58(0.33-1.02)$ & 0.33 & 40.0 & 87.3 \\
VAP-I & $0.54(0.37-0.70)$ & 0.43 & 0.47 & 0.70 & $1.56(0.8-3.02)$ & $0.76(0.46-1.26)$ & 0.17 & 28.0 & 84.0 \\
IL-6 & $0.69(0.54-0.85)$ & 0.24 & 0.53 & 0.85 & $3.56(1.65-7.65)$ & $0.55(0.32-0.95)$ & 0.38 & 47.1 & 87.9 \\
\hline
\end{tabular}

Note: Sensitivity, specificity, positive and negative predictive values were calculated using optimal cut-off values determined with Youden index. EG: endothelial glycocalyx; sTM: soluble thrombomodulin (pg/L); syndecan-I (ng/L); VAP-I: vascular adhesion protein I (ng/L); IL-6: interleukin-6 (pg/L); PMI: perioperative myocardial infarction; AUC: area under receiver operating characteristics curve; $\mathrm{Cl}$ : confidence interval; $\mathrm{LR}+:$ positive likelihood ratio; LR-: negative likelihood ratio; PPV: positive predictive value; NPV: negative predictive value. 
demonstrated that plasma syndecan-1 and heparan sulphate concentrations rose in patients undergoing procedures predisposing them to either global or regional ischaemia followed by reperfusion, whereas intercellular adhesion molecule-1 (ICAM-1) and vascular cell adhesion molecule-1 (VCAM-1) concentrations remained unchanged. ${ }^{9}$ This finding led the authors to the conclusion that the circulating components of EG may be more sensitive markers of EG injury than integral membrane proteins. Furthermore, Rehm et al. observed a high interindividual variation in EG shedding, which is in broad agreement with our results. The differences in surgical procedures, perioperative management and the patients' medical condition are a plausible explanation for the variation.

Despite the great emphasis on perioperative care during the past years, PMI remains a severe complication, with an in-hospital mortality of $12 \%-40 \%{ }^{28-31}$ Perioperative hemodynamic instability, leading to oxygen demand/supply disparity, as well as blood flow stagnation and thrombus formation are regarded as major factors in the development of PMI. ${ }^{32}$ Therefore, most trials have been targeted towards preventing these adverse factors. However, despite numerous large-scale clinical studies, ${ }^{33-35}$ efficient and safe methods to prevent PMI are still lacking, and it is likely that we remain unaware of the exact pathophysiological mechanisms of PMI. Considering the physiological disturbances - with impaired vasodilatation, enhanced plaque vulnerability and pro-thrombotic state in particular - caused by a dysfunctional endothelium, endothelial injury seems a logical contributor in the development of PMI. Furthermore, several studies have demonstrated an association between the presence of coronary endothelial dysfunction and ischaemic symptoms or imaging findings in individuals without clinically significant coronary artery disease. ${ }^{36-39}$ This might explain the fact that perioperative cardiac complications are sometimes hard to predict and may occur in patients with relatively clean coronary arteries. ${ }^{40}$

To the best of our knowledge, this was the first study to suggest that EG injury may play a role in the pathophysiology of PMI, and this finding opens the door for possible future therapeutic trials. An interesting report published by Manchurov et al. demonstrated that remote ischaemic preconditioning (RIPC) before pPCI clearly improved the endothelial function in patients with an acute myocardial infarction. ${ }^{41}$ Whether similar effects can be achieved in surgical patients and whether this is beneficial in preventing perioperative cardiac complications and improving perioperative outcome could be a subject of future clinical trials. In order to conduct these trials, though, larger observational studies on the association between
EG injury and perioperative cardiac complications are needed.

The current study has some important limitations. Firstly, because of the missing blood samples, we were not able to include all consecutive patients of the primary study and, unfortunately, had to exclude $12 \mathrm{PMI}$ patients. The current results should be interpreted taking this into consideration. However, as presented in Tables S1 and S2, patients with and without all blood samples available were comparable regarding their baseline characteristics. Additionally, we matched controls for the PMI patients using a propensity score and increased the statistical power by including four controls for each PMI patient. Secondly, this was an observational study, not allowing an evaluation of the causal relationship. Finally, the assessment of diagnostic ability (using positive likelihood ratios) was based on cut-off values derived from the same study because previous values for PMI patients did not exist in the literature. Despite the small sample size, there was a fairly strong correlation between cTnT, IL-6 and EG markers in 15 PMI patients. Thus, our findings should be considered as first hypothesis-generating preliminary evidence regarding the potential association between EG injury and PMI.

\section{Conclusion}

Systemic inflammation, reflected by IL-6, associates with cTnT release and PMI. Furthermore, IL-6 demonstrated some potential to predict PMI, whereas EG markers did not. These findings should be considered preliminary and need to be confirmed or refuted in larger future studies.

\section{Acknowledgements}

We want to thank research assistants Riikka Kosonen and Sanni Perttunen for their valuable assistance with the ELISA analyses.

\section{Declaration of conflicting interests}

The author(s) declared no potential conflicts of interest with respect to the research, authorship, and/or publication of this article.

\section{Funding}

The author(s) disclosed receipt of the following financial support for the research, authorship, and/or publication of this article: The study was supported by clinical research funding no. TYH 2014239 from the University of Helsinki and Helsinki University Hospital (VP, LV) and by grants from the Sigrid Juselius foundation (VP) and the Finnish Society of Angiology (LV).

\section{Ethical approval}

Ethical approval for the study (no. 11/13/03/02/2014) was provided by the Ethics Committee of the Department of Surgery at Helsinki University Hospital, Helsinki, Finland, on 30 January 2014.

\section{Guarantor}

VP 


\section{Contributorship}

AO, LV and VP contributed to the design of the study. AO conducted literature searches, gathered clinical data, wrote the first draft and edited the final version of the paper. JV made all the PMI diagnoses. PL was responsible for the laboratory analyses. AO performed all statistical analyses, and SN acted as a consultant. All of the authors contributed to the critical review of the article and approved the final version.

\section{ORCID iD}

Aino Ollila (D) https://orcid.org/0000-0002-6845-8654

\section{Supplemental material}

Supplemental material for this article is available online.

\section{References}

1. Wang L, Fuster M, Sriramarao P, et al. Endothelial heparan sulfate deficiency impairs L-selectin- and chemokine-mediated neutrophil trafficking during inflammatory responses. Nat Immunol 2005; 6: 902-910.

2. Platts SH, Linden $\mathrm{J}$ and Duling BR. Rapid modification of the glycocalyx caused by ischemia-reperfusion is inhibited by adenosine A2A receptor activation. Am J Physiol Heart Circ Physiol 2003; 284: H2360-H2367.

3. Kurzelewski M, Czarnowska E and Beresewicz A. Superoxide- and nitric oxide-derived species mediate endothelial dysfunction, endothelial glycocalyx disruption, and enhanced neutrophil adhesion in the post-ischemic guinea-pig heart. J Physiol Pharmacol 2005; 56: 163-178.

4. Mulivor AW and Lipowsky HH. Inflammation- and ischemia-induced shedding of venular glycocalyx. Am J Physiol Heart Circ Physiol 2004; 286: H1672-H1680.

5. Ostrowski SR, Gaini S, Pedersen C, et al. Sympathoadrenal activation and endothelial damage in patients with varying degrees of acute infectious disease: an observational study. J Crit Care 2015; 30: 90-96.

6. Bashandy GM. Implications of recent accumulating knowledge about endothelial glycocalyx on anesthetic management. J Anesth 2015; 29: 269-278.

7. Gabay C. Interleukin-6 and chronic inflammation. Arthritis Res Ther 2006; 8: S3.

8. Johansen ME, Johansson PI, Ostrowski SR, et al. Profound endothelial damage predicts impending organ failure and death in sepsis. Semin Thromb Hemost 2015; 41: 16-25.

9. Rehm M, Bruegger D, Christ F, et al. Shedding of the endothelial glycocalyx in patients undergoing major vascular surgery with global and regional ischemia. Circulation 2007; 116: 1896-1906.

10. Ostrowski SR, Pedersen SH, Jensen JS, et al. Acute myocardial infarction is associated with endothelial glycocalyx and cell damage and a parallel increase in circulating catecholamines. Crit Care 2013; 17: R32.

11. Bonetti PO, Lerman LO and Lerman A. Endothelial dysfunction: a marker of atherosclerotic risk. Arterioscler Thromb Vasc Biol 2003; 23: $168-175$.

12. Gokce N. Clinical assessment of endothelial function: ready for prime time? Circ Cardiovasc Imaging 2011; 4: 348-350.

13. Suwaidi JA, Hamasaki S, Higano ST, et al. Long-term follow-up of patients with mild coronary artery disease and endothelial dysfunction. Circulation 2000; 101: 948-954.

14. Ollila A, Vikatmaa L, Virolainen J, et al. Perioperative myocardial infarction in non-cardiac surgery patients: a prospective observational study. Scand J Surg 2017; 106: 180-186.

15. Thygesen K, Alpert JS, Jaffe AS, et al. Third universal definition of myocardial infarction. J Am Coll Cardiol 2012; 60: 1581-1598.

16. Ray P, Le Manach Y, Riou B, et al. Statistical evaluation of a biomarker. Anesthesiology 2010; 112: 1023-1040.

17. Gupta PK, Gupta H, Sundaram A, et al. Development and validation of a risk calculator for prediction for cardiac risk after surgery. Circulation 2011; 124: 381-387.
18. Ross R. Atherosclerosis - an inflammatory disease. N Engl J Med 1999; 340: 115-126.

19. Kinlay S and Ganz P. Role of endothelial dysfunction in coronary artery disease and implications for therapy. Am J Cardiol 1997; 80: 11I-16I

20. Libby P. Current concepts of the pathogenesis of the acute coronary syndromes. Circulation 2001; 104: 365-372.

21. Becker BF, Chappell D, Bruegger D, et al. Therapeutic strategies targeting the endothelial glycocalyx: acute deficits, but great potential. Cardiovasc Res 2010; 87: 300-310.

22. Bro-Jeppesen J, Johansson PI, Hassager C, et al. Endothelial activation/ injury and associations with severity of post-cardiac arrest syndrome and mortality after out-of-hospital cardiac arrest. Resuscitation 2016; 107: 71-79.

23. Ohlin AK, Larsson K and Hansson M. Soluble thrombomodulin activity and soluble thrombomodulin antigen in plasma. J Thromb Haemost 2005; 3: 976-982.

24. Bruegger D, Jacob M, Rehm M, et al. Atrial natriuretic peptide induces shedding of endothelial glycocalyx in coronary vascular bed of guinea pig hearts. Am J Physiol Heart Circ Physiol 2005; 289: H1993-H1999.

25. Chappell D, Jacob M, Hofmann-Kiefer K, et al. Hydrocortisone preserves the vascular barrier by protecting the endothelial glycocalyx. Anesthesiology 2007; 107: 776-784.

26. Sallisalmi M, Tenhunen J, Yang R, et al. Vascular adhesion protein-1 and syndecan-1 in septic shock. Acta Anaesthesiol Scand 2012; 56: 316-322.

27. Salmi M, Stolen C, Jousilahti P, et al. Insulin-regulated increase of soluble vascular adhesion protein-1 in diabetes. Am $J$ Pathol 2002; 161: 2255-2262.

28. Devereaux PJ, Goldman L, Yusuf S, et al. Surveillance and prevention of major perioperative ischemic cardiac events in patients undergoing noncardiac surgery: a review. CMAJ 2005; 173: 779-788.

29. Devereaux PJ, Xavier D, Pogue J, et al. Characteristics and short-term prognosis of perioperative myocardial infarction in patients undergoing noncardiac surgery: a cohort study. Ann Intern Med 2011; 154: 523-528.

30. Davenport DL, Ferraris VA, Hosokawa P, et al. Multivariable predictors of postoperative cardiac adverse events after general and vascular surgery: results from the patient safety in surgery study. J Am Coll Surg 2007; 204: 1199-1210.

31. Ghaferi AA, Birkmeyer JD and Dimick JB. Variation in hospital mortality associated with inpatient surgery. N Engl J Med 2009; 361: 1368-1375.

32. Biccard BM and Rodseth RN. The pathophysiology of peri-operative myocardial infarction. Anaesthesia 2010; 65: 733-741.

33. POISE Study Group, Devereaux PJ, Yang H, Yusuf S, et al. Effects of extended-release metoprolol succinate in patients undergoing non-cardiac surgery (POISE trial): a randomised controlled trial. Lancet 2008; 371: 1839-1847.

34. Devereaux PJ, Mrkobrada M, Sessler DI, et al. Aspirin in patients undergoing noncardiac surgery. N Engl J Med 2014; 370: 1494-1503.

35. Devereaux PJ, Sessler DI, Leslie K, et al. Clonidine in patients undergoing noncardiac surgery. $N$ Engl J Med 2014; 370: 1504-1513.

36. Egashira K, Inou T, Hirooka Y, et al. Evidence of impaired endotheliumdependent coronary vasodilatation in patients with angina pectoris and normal coronary angiograms. N Engl J Med 1993; 328: 1659-1664.

37. Motz W, Vogt M, Rabenau O, et al. Evidence of endothelial dysfunction in coronary resistance vessels in patients with angina pectoris and normal coronary angiograms. Am J Cardiol 1991; 68: 996-1003.

38. Quyyumi AA, Cannon RO, Panza JA, et al. Endothelial dysfunction in patients with chest pain and normal coronary arteries. Circulation 1992; 86: $1864-1871$.

39. Zeiher AM, Krause T, Schächinger V, et al. Impaired endotheliumdependent vasodilation of coronary resistance vessels is associated with exercise-induced myocardial ischemia. Circulation 1995; 91: 2345-2352.

40. Sheth T, Chan M, Butler C, et al. Prognostic capabilities of coronary computed tomographic angiography before non-cardiac surgery: prospective cohort study. BMJ 2015; 350: h1907.

41. Manchurov V, Ryazankina N, Khmara T, et al. Remote ischemic preconditioning and endothelial function in patients with acute myocardial infarction and primary PCI. Am J Med 2014; 127: 670-673. 